\title{
Corrigendum: The Nrf2-Antioxidant Response Element Signaling Pathway Controls Fibrosis and Autoimmunity in Scleroderma
}

\author{
Niloufar Kavian ${ }^{1,2}$, Souad Mehlal, ${ }^{1,2}$, Mohamed Jeljeli ${ }^{1,2}$, Nathaniel Edward Bennett Saidu ${ }^{2}$, \\ Carole Nicco ${ }^{2}$, Olivier Cerles ${ }^{2}$, Sandrine Chouzenoux ${ }^{2}$, Anne Cauvet ${ }^{2}$, Claire Camus ${ }^{2}$, \\ Mehdi Ait-Djoudi ${ }^{2}$, Christiane Chéreau ${ }^{2}$, Saadia Kerdine-Römer ${ }^{3}$, Yannick Allanore ${ }^{2,4}$ \\ and Frederic Batteux ${ }^{1,2 *}$ \\ ${ }^{1}$ Laboratoire d'Immunologie, Hôpital Cochin, Paris, France, 2 INSERM U1016, Institut Cochin, Paris, France, ${ }^{3}$ UMR996 - \\ Inflammation, Chemokines and Immunopathology, INSERM, Univ Paris-Sud, Université Paris-Saclay, Châtenay-Malabry, \\ France, ${ }^{4}$ Service de Rhumatologie, Hôpital Cochin, Paris, France
}

Keywords: systemic sclerosis, oxidative stress, fibrosis, inflammation, Nrf2

\section{OPEN ACCESS}

Edited and reviewed by: Danièle Noël, U1183 Cellules Souches, Plasticité Cellulaire, Médecine Régénératrice Et Immunothérapies (INSERM), France

*Correspondence: Frederic Batteux frederic.batteux@aphp.fr

Specialty section:

This article was submitted to Immunological Tolerance and Regulation,

a section of the journal

Frontiers in Immunology

Received: 06 July 2021 Accepted: 12 July 2021 Published: 27 July 2021

Citation:

Kavian N, Mehlal S, Jeljeli M, Saidu NEB, Nicco C, Cerles O, Chouzenoux S, Cauvet A, Camus $C$, Ait-Djoudi M, Chéreau C, Kerdine-Römer S, Allanore $Y$ and Batteux F (2021) Corrigendum: The Nrf2-Antioxidant Response Element Signaling Pathway Controls Fibrosis and Autoimmunity in Scleroderma.

Front. Immunol. 12:737303. doi: 10.3389/fimmu.2021.737303

\section{A Corrigendum on}

The Nrf2-Antioxidant Response Element Signaling Pathway Controls Fibrosis and Autoimmunity in Scleroderma

By Kavian N, Mehlal S, Jeljeli M, Saidu NEB, Nicco C, Cerles O, Chouzenoux S, Cauvet A, Camus C, Ait-Djoudi M, Chéreau C, Kerdine-Römer S, Allanore Y and Batteux F (2018). Front. Immunol. 9:1896. doi: 10.3389/fimmu.2018.01896

In the original article, there was a mistake in the legend for Figure 3G and $\mathbf{6} \mathbf{I}$ as published. Skin and lung biopsies were stained with Haematoxylin and Eosin, and not "with picro-sirius red and Haematoxylin and Eosin" as stated in the original legend. The correct legends appear below. The authors apologize for this error and state that this does not change the scientific conclusions of the article in any way. The original article has been updated.

In the original article, there was a mistake in the photos used in Figures $\mathbf{3 G}$ and $\mathbf{6 I}$ as published. The authors noticed that some of the lung histology photos used in Figures $\mathbf{3}$ and $\mathbf{6}$ as illustrations only were not correct due to an inadvertent mishandling of the names list and photo files. The corrected Figures $\mathbf{3}$ and $\mathbf{6}$ appear below. The authors apologize for this error and state that this does not change the scientific conclusions of the article in any way, as these photos were used as illustrations only, and not as a source of quantifiable data. The original article has been updated.

In the original article, there was an error in the Methods section: "A $5-\mu \mathrm{m}$-thick tissue section was prepared from the mid-portion of paraffin-embedded tissue and stained with H\&E or picro-sirius red."

A correction has been made in the text of the Methods section, Assessment of Skin Thickness and Collagen Accumulation in Skin and Lungs, paragraph 1:

"A 5- $\mu \mathrm{m}$-thick tissue section was prepared from the mid-portion of paraffin-embedded tissue and stained with H\&E."

In the original article, there was an error in the Results section: "Staining of skin and lung biopsies with picro-sirius red also showed a reduction in fibrosis in both organs in diseased-mice treated with DMF compared to untreated diseased-mice (Figure 6I)." 
G

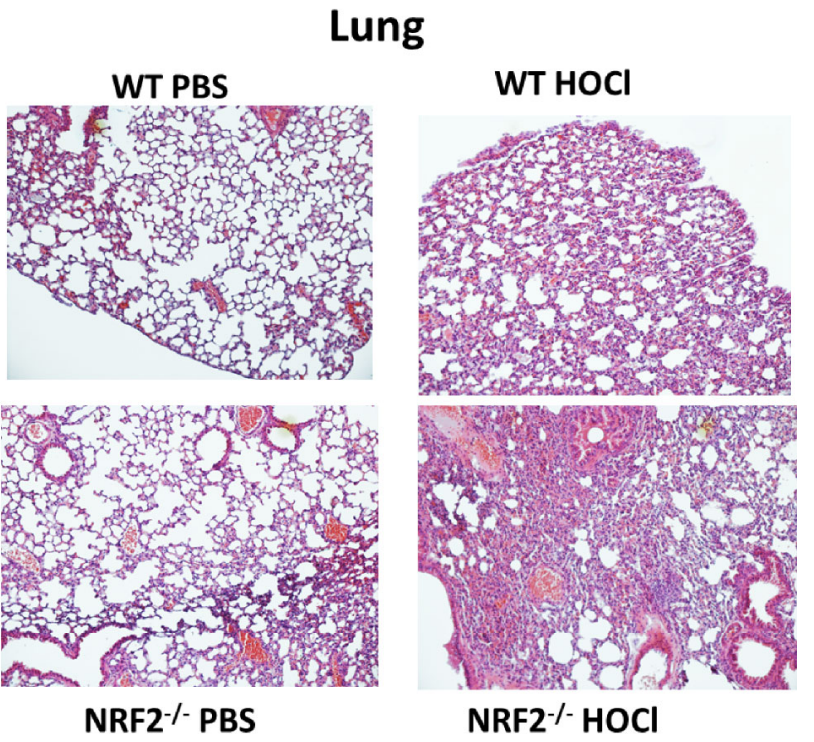

FIGURE 3G | (F, G) Skin and Lung biopsies stained with Hematoxylin and eosin (H\&E).

I

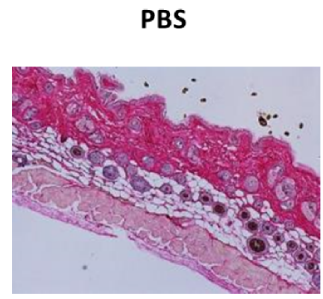

PBS+DMF

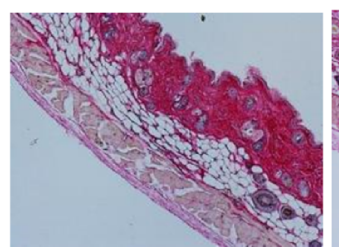

HOCl

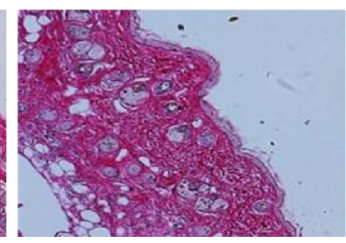

HOCl+DMF

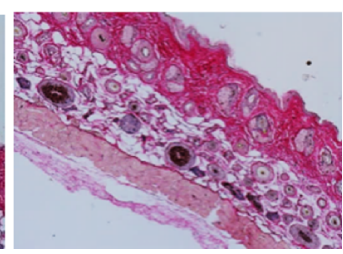

PBS

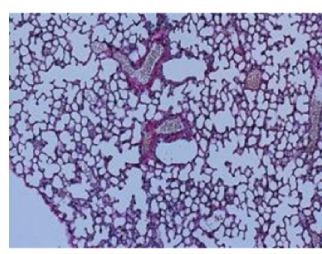

PBS+DMF

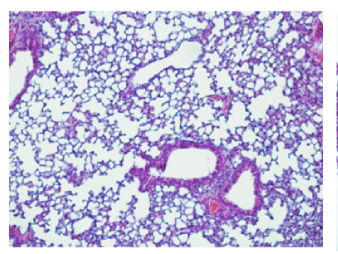

$\mathrm{HOCl}$

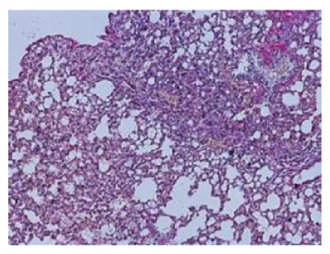

HOCl+DMF

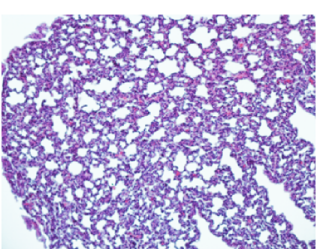

FIGURE 6I | (I) Skin and lung biopsies stained with Hematoxylin and eosin (H\&E).

A correction has been made in the text of the Results section, Treatment of HOCl-mice with DMF prevents the development of SSc, paragraph 1:

"Staining of skin and lung biopsies with Hematoxylin and Eosin also showed a reduction in fibrosis in both organs in diseased-mice treated with DMF compared to untreated diseased-mice (Figure 6I).”

The authors apologize for this error and state that this does not change the scientific conclusions of the article in any way. The original article has been updated.
Publisher's Note: All claims expressed in this article are solely those of the authors and do not necessarily represent those of their affiliated organizations, or those of the publisher, the editors and the reviewers. Any product that may be evaluated in this article, or claim that may be made by its manufacturer, is not guaranteed or endorsed by the publisher.

Copyright (C) 2021 Kavian, Mehlal, Jeljeli, Saidu, Nicco, Cerles, Chouzenoux, Cauvet, Camus, Ait-Djoudi, Chereau, Kerdine-Römer, Allanore and Batteux. This is an openaccess article distributed under the terms of the Creative Commons Attribution License (CC BY). The use, distribution or reproduction in other forums is permitted, provided the original author(s) and the copyright owner(s) are credited and that the original publication in this journal is cited, in accordance with accepted academic practice. No use, distribution or reproduction is permitted which does not comply with these terms. 